\title{
NOTICIAS DE LA FACULTAD
}

\section{Proyecto Tarapacá}

Texto: Umberto Bonomo, equipo ganador concurso Tarapacá

Felipe Kramm, Grupo Tarapacá

Proyecto Tarapacá es una iniciativa liderada por estudiantes de pregrado y posgrado de la Escuela de Arquitectura de la Pontificia Universidad Católica de Chile, que a través de la investigación, reflexión y acción respecto de la situación del patrimonio cultural y arquitectónico de los poblados del extremo norte de Chile, pretende aportar a la discusión sobre la intervención y reconstrucción en contextos patrimoniales.

El proyecto propone una postura distinta frente a estos temas, entendiendo que el valor del patrimonio arquitectónico no está radicado sólo en los monumentos, sino también en el tejido urbano y la estructura y espacialidad de las viviendas tradicionales populares.

La iniciativa nace en respuesta al terremoto grado 7,8 en la escala de Richter ${ }^{1}$ que azotó el 17 de junio de 2005 a la región de Tarapacá, destruyendo gran parte de los poblados ubicados en las quebradas al interior. Un grupo de estudiantes de la Escuela de Arquitectura de la Pontificia Universidad Católica de Chile acogió el llamado del Ministerio de Vivienda y Urbanismo para hacer un registro de los daños provocados por dicho terremoto, realizando un minucioso análisis con el fin de identificar los aspectos patrimoniales relevantes del pueblo de San Lorenzo de Tarapacá -en la provincia de Iquique- y determinar el estado en que se encontraban sus viviendas. Una vez realizado este trabajo se formó Proyecto Tarapacá: el objetivo específico de este proyecto es la recuperación de las viviendas mediante sistemas de edificación sustentables, económicos y resistentes que permitan la construcción de hogares dignos y respetuosos del patrimonio local. Se propone además buscar estrategias de intervención posibles de aplicar en el sector público.

La labor realizada por el grupo se concentró en temas de habitabilidad ligados a condiciones culturales, religiosas y climáticas locales, aspectos fundamentales para el desarrollo de propuestas que integraran valores patrimoniales. Luego de este estudio, durante 2006 se organizó un concurso a nivel nacional para estudiantes universitarios, buscando definir lineamientos para intervenir en poblados devastados por catástrofes naturales. A partir del desarrollo de un prototipo de unidad habitacional, debían hacerse cargo tanto de las problemáticas urbanas como de las constructivas, proponiendo nuevas técnicas y materialidades en respuesta a las condiciones climáticas del desierto chileno. El prototipo de la vivienda debía procurar un doble concepto de eficiencia: velocidad de construcción y adaptabilidad a distintos emplazamientos. Se recibieron 22 proyectos provenientes de distintas partes de
Chile, evaluadas por un jurado compuesto por profesionales, académicos y autoridades ${ }^{2}$.

La propuesta ganadora fue la del equipo conformado por los arquitectos y estudiantes del programa de Doctorado en Arquitectura y Estudios Urbanos Puc Phillippe Blanc y Umberto Bonomo, el arquitecto Cristián Olivos y los estudiantes de la Escuela de Arquitectura Puc Macarena Burdiles y Juan Eduardo Ojeda. Ellos plantean reconstruir a través de la vivienda dos elementos centrales en la ordenación del lugar: el trazado urbano y la fachada continua destruida por el terremoto, ya que es en las calles y en los espacios públicos que cada año se efectúan las fiestas en honor al patrono San Lorenzo, parte fundamental del patrimonio cultural y social del lugar.

La unidad proyectada reinterpreta el perfil de la calle con un zócalo continuo -conformado en parte por los restos dejados por el terremoto- y la sombra de un alero de cañas, característico de la arquitectura vernácula del norte de Chile. Esta propuesta infraestructural, vinculada de las características geoclimáticas del lugar, responde satisfactoriamente a las costumbres y formas de vida de los habitantes.

La casa muro, como se le denominó para el concurso, se conforma por corchetes modulares de $3 \mathrm{x}$ $1,5 \mathrm{~m}$, cuya flexibilidad permite generar soluciones distributivas para una variedad de viviendas. Se utilizan ladrillos fiscales pintados con cal y tra-

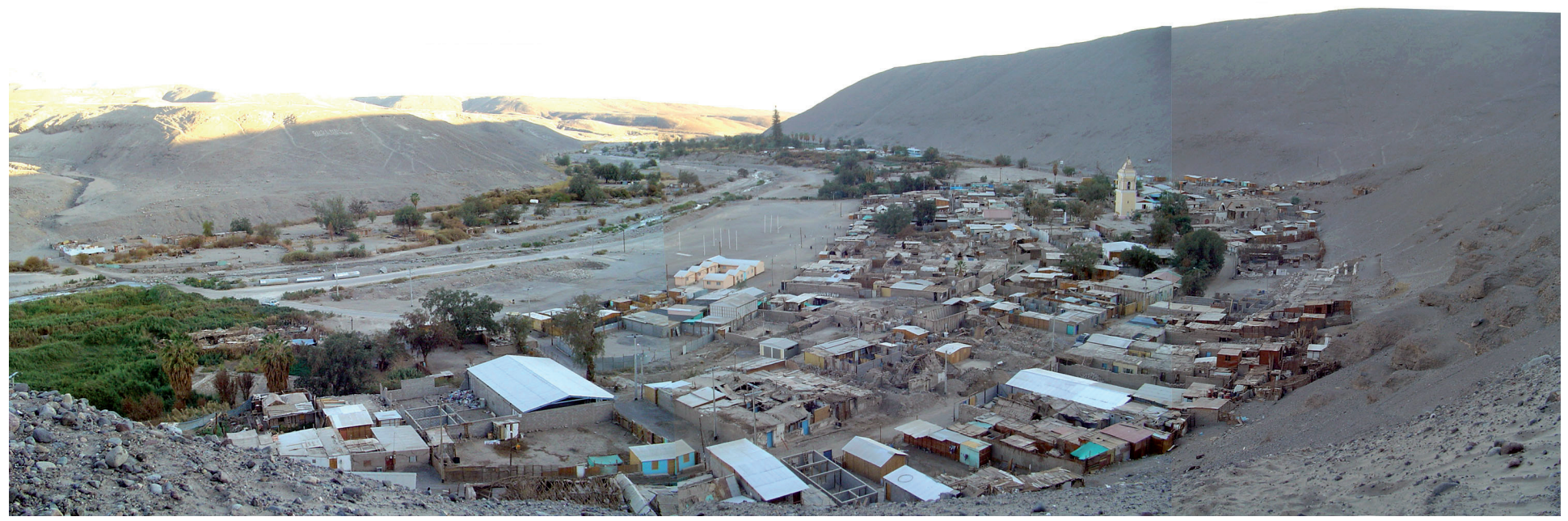

San Lorenzo de Tarapacá después del terremoto del 17 de junio de 2005 
1 Jurado del concurso: José Rosas (Decano Facultad de Arquitectura, Diseño y Estudios Urbanos puc), Fernando Pérez Oyarzún (Jefe programa de Doctorado en Arquitectura y Estudios Urbanos puc), Khaled Pascha (docente Technische Universität Berlin), Marcelo Huenchuñir (docente Universidad de Chile y Universidad Andrés Bello), Javier del Rio versidad Andrés Bello), Javier del Rĩo (docente Escuela de Arquitectura puc y Universidad Andrés Bello), Nicolás Rebolledo (Cooperativa URO1.ORC), Francisco Prado (docente Escuela de Construcción Civil puc), Paula Leyton (arquitecto mivvu Tarapać) y Héctor López (arquitecto DITEC-MINvU).

$$
\begin{aligned}
& 2 \text { Fuente: Servicio Sismológico de } \\
& \text { la Universidad de Chile, http://ssn. }
\end{aligned}
$$
dgf.uchile.cl bados entre sí, dándole mayor resistencia al muro y generando un volumen de aire contenido capaz de aumentar el aislamiento térmico y acústico. Durante el mes de enero de 2007 los ganadores del concurso, en conjunto con el Grupo Tarapacá, desarrollaron un prototipo constructivo para albergar una biblioteca y una sala multiusos administrada por la Junta de Vecinos. La ejecución de su primera etapa -una oficina de $9 \mathrm{~m} 2-$ se realizó en febrero del mismo año, con la participación del Grupo Tarapacá, voluntarios y pobladores del lugar. Al concretar el prototipo, se puso a prueba el sistema constructivo desarrollado frente a los pobladores y las autoridades como una alternativa de reconstrucción sencilla, económica, antisísmica, respetuosa de las condiciones patrimoniales y consecuente con la forma de habitar en zonas áridas. La propuesta logra alta inercia térmica mezclando técnicas y productos industrializados ya empleados localmente y materiales propios del lugar. Los muros están compuestos por una estructura portante de albañilería armada de bloques de hormigón, cubierta en su cara exterior con bloques de adobe en pandereta recuperados del terremoto. Las distintas capas, de función específica, forman un muro de $36 \mathrm{~cm}$ de espesor capaz de mediar con las extremas condiciones climáticas del desierto logrando que la temperatura de los recintos se mantenga tanto en el día como en la noche. La implementación de técnicas locales en la cubierta resulta indispensable para evitar el sobrecalentamiento del recinto; se dispuso un techo ventilado entre la cubierta de zinc aluminio y el cielo de cañas y barro.

El diseño arquitectónico y la distribución de los módulos que conforman el proyecto para la sede social enfatizan la importancia de los espacios intermedios: los patios y pasillos, normalmente entendidos como resultantes entre los volúmenes construidos, median el paso entre interior y exterior. Sus cubiertas, construidas con materiales del lugar, controlan la fuerte radiación solar.

El prototipo Tarapacá propone un sistema constructivo replicable y económico, que se ajusta fácilmente a las múltiples formas de parcelación existentes en el poblado de San Lorenzo de Tarapacá y que puede transformarse en un motor ágil para su reconstrucción.

+ info: www.proyectotarapaca.org

Grupo Tarapacá Samuel Bravo, Bernardita Devilat, Verónica Illanes, Felipe Kramm, Álvaro Silva, Natalia Spörke

Desarrollo del prototipo Philippe Blanc, Umberto Bonomo, Samuel Bravo, Macarena Burdiles, Bernardita Devilat, Verónica Illanes, Cristián Olivos, Álvaro Silva, Natalia Spörke, Felipe Kramm

Colaboradores Renato D'Alençon, Francisco Prado

Construcción Proyecto Tarapacá (Samuel Bravo, Bernardita Devilat Verónica Illanes, Felipe Kramm, Álvaro Silva, Natalia Spörke, Francisco Prado), voluntarios (Carlos Castro, Valeria Carrasco, Cristián Cciolina Daniela Leiva, Javier Muñoz, José Manuel Pérez, Jennifer Quiroz Valeria Razeto, Juan Pablo Torrealba), Ejército de Chile y pobladores de San Lorenzo de Tarapacá

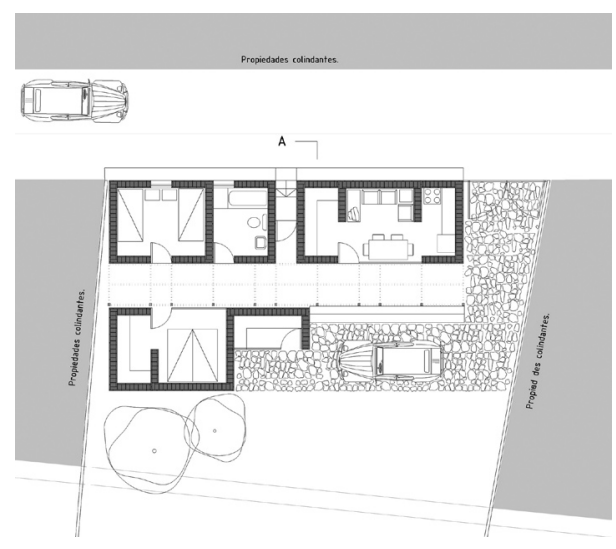

Proyecto ganador del concurso, hipótesis de configuración planimétrica

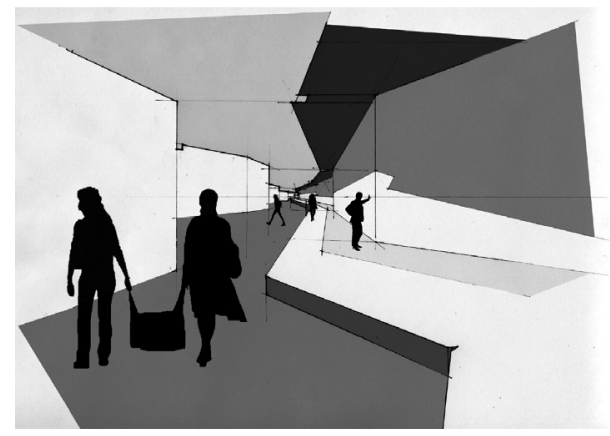

Proyecto ganador del concurso, imagen del espacio público

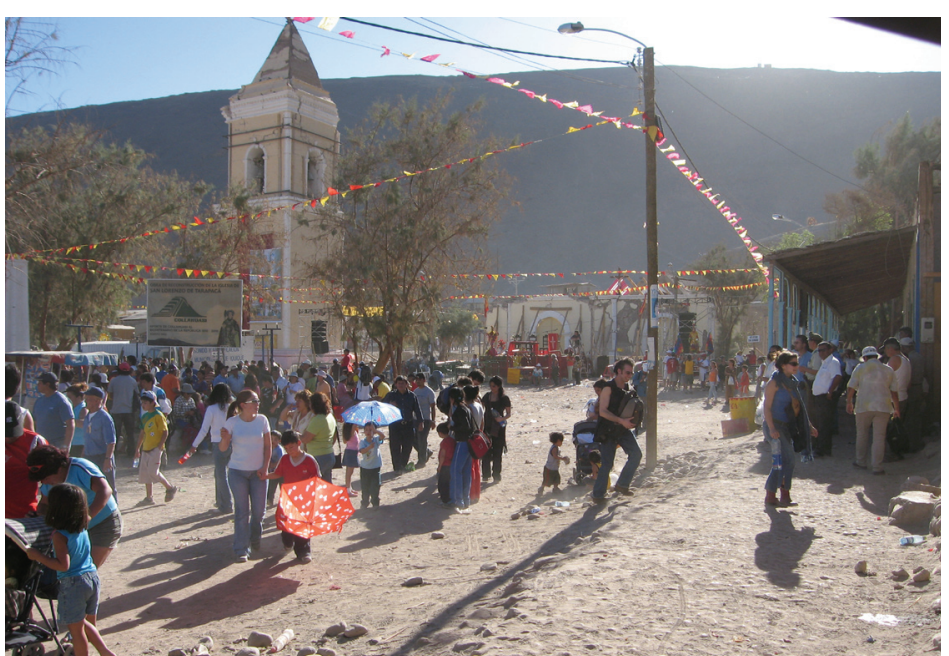

Fiesta de San Lorenzo. Fotografía de Álvaro Silva

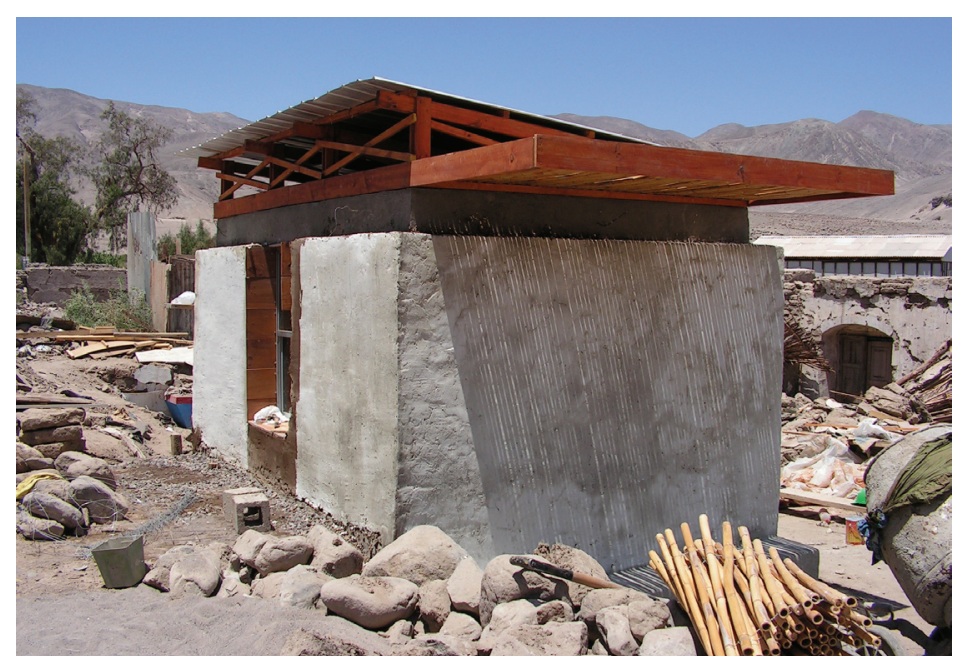

Prototipo construido en febrero de 2007. Fotografía de Bernardita Devilat 